\title{
MouseBank: A Database Application for Managing Transgenic Mouse Breeding Programs
}

BioTechniques 30:130-132 (January 2001)

\section{Richard Hopley ${ }^{1}$ and Andreas Zimmer ${ }^{1,2}$ \\ INational Institute of Mental Health, National Institutes of Health, Bethesda, MD, USA; ${ }^{2}$ University of Bonn, Bonn, Germany}

We have developed a powerful tool for managing transgenic and knockout mouse programs using the Macintosh ${ }^{\circledR}$ and Microsoft ${ }^{\circledR}$ Windows ${ }^{\mathrm{TM}}$-compatible relational database management system, 4th Dimension ${ }^{\circledR}$ (4D). MouseBank is a user-friendly system designed for small laboratories and large workgroups to organize every aspect of the generation and breeding of transgenic animals.

MouseBank is built on the client/ server model to handle any arbitrarily large number of researchers and projects. Using MouseBank, each researcher manages his or her own projects from a desktop computer, while the database resides on a fast server. MouseBank has been tested and is currently in use on Macintosh computers.

MouseBank offers two views of the data. The Lab Director View is a traditional table-oriented view of the seven interrelated project data tables: Project, Mating Pair, Litter, DNA Construct, ES Clone, Production Experiment and Mouse. Records in an eighth table, User, are created by the database administrator and are used to enforce password-controlled access to the data (several other tables, largely invisible to the user, store database data as opposed to breeding project data). In the $\mathrm{Re}$ searcher View, researchers can view only the records relating to the projects for which they are designated PI or to which they have been authorized access by the PI. Researchers are guided strictly through the logical hierarchy of the tables. Most users are restricted to the Researcher View; the Lab Director View, which cuts across all user boundaries, provides a cumulative overview of all breeding colonies in the laboratory to a few authorized users.

Several program features minimize manual data entry. For example, mice are identified by any (up to) 16 characters, but if the ID contains a block of successive numerals, then the computer helps with the data entry. When a new litter is born, the researcher enters date of birth, location and strain of the litter and the ID of the first mouse. Thereafter, these data are stamped onto each successive pup record created during that session, incrementing the numeric part of the ID so that the researcher only enters the sex and genotype of subsequent pups in the litter. The user can override these computer-generated IDs at any time, however.

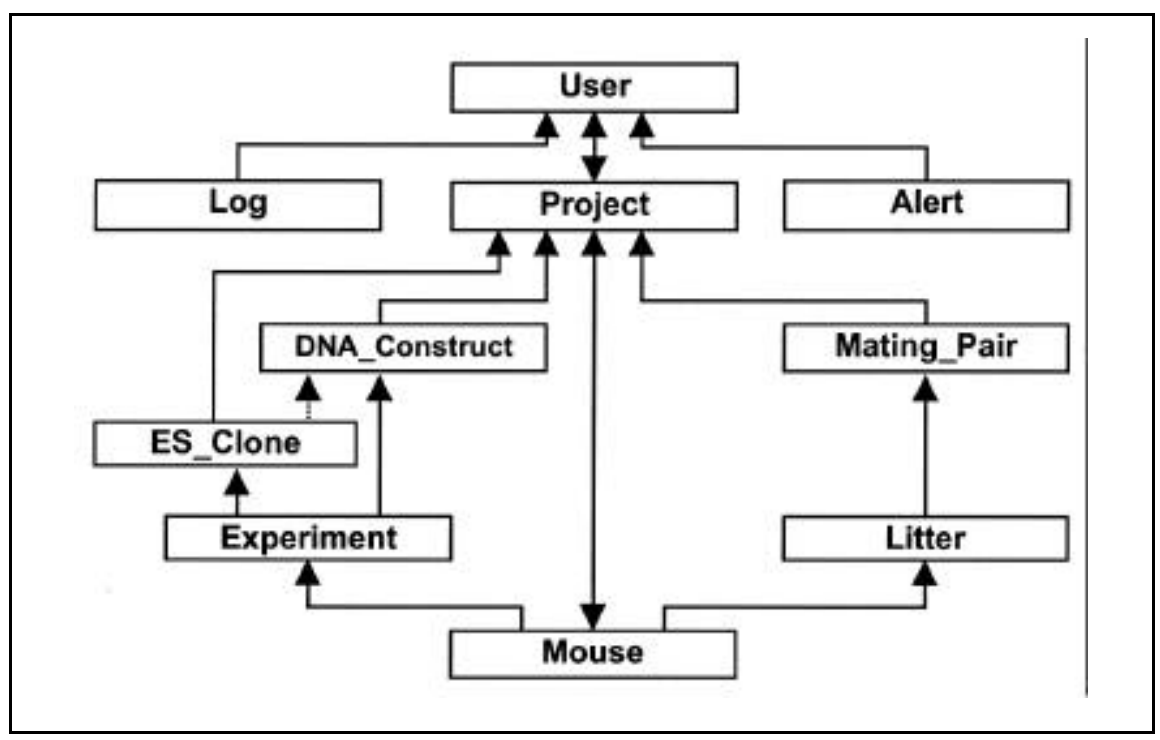

Figure 1. Structure and relationships of MouseBank 2.1 tables. Shown are all tables directly visible to the user. Arrowheads identify relationships as many-to-one or many-to-many. In a many-to-one relationship, as between Mouse and Litter, an arbitrary number of mice may belong to a litter, but only one litter can produce a given mouse. In a many-to-many relationship, as between Project and Mouse, many mice will be used in one project, but one mouse may also be used in many projects. The dependency of ES Clone on DNA Construct is not enforced by the program to allow the use of clones from outside sources that were not made from constructs developed in this laboratory. 


\section{$\gg \gg \gg>\gg>>>>>>>>>$}

To further reduce the time and to im prove the accuracy of data entry, each user can create choice lists of mouse strains, genotypes and other restricted value sets. These individual choice lists can be tailored to the need of each pro- ject and allow data selection from concise lists with "double-clicks".

The program updates the ages (in days and in weeks) of living mice daily and can prepare a genealogy of any mouse as needed or a printed report on



Figure 2. Main menu at program entrypoint: the Researcher View. A password dialog determines the user and displays the User record. In the menu, where the database administrator has granted Lab Director privileges, the Lab Director View option is enabled. If Administrator privileges are granted, then Administration and System Setup options are enabled. Tab controls in the User record show related records and other data fields. When the Projects tab is foremost, users can see all projects to which they have access. Where he or she is the PI, the user has full privileges; otherwise, he or she may have been granted lesser privileges.

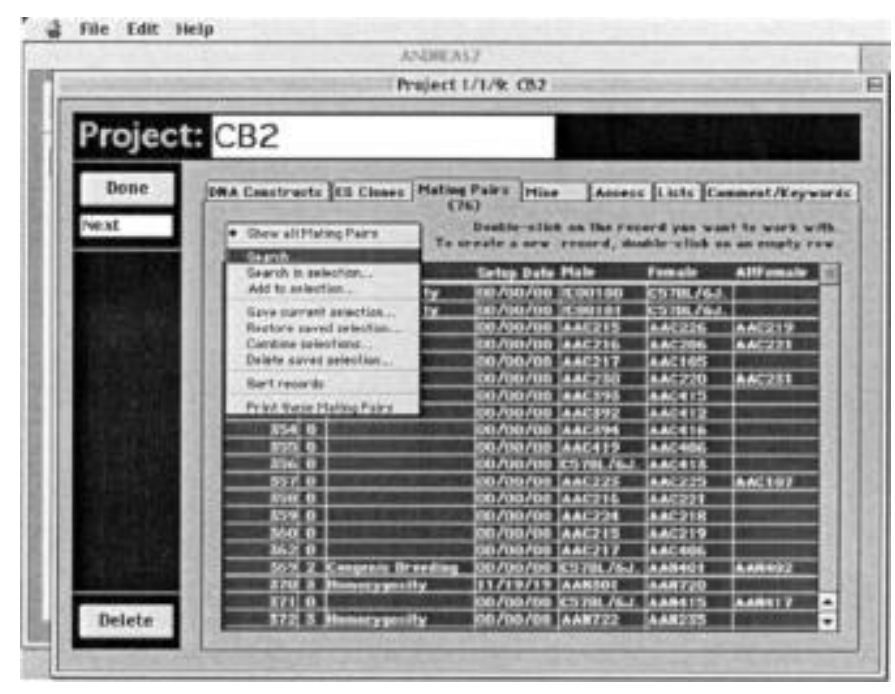

Figure 3. A Project record selected from User display including pop-up menu. For each of the seven project data tables, tab controls display related records or other fields. Each data table includes a $32000-$ character text field for comments and a keyword list to facilitate record retrieval. Each display includes a pop-up menu of tools for operating upon the related records in the included table. Here, the option "Show All Mating Pairs" displays all Mating Pair records related to the currently displayed Project record. 


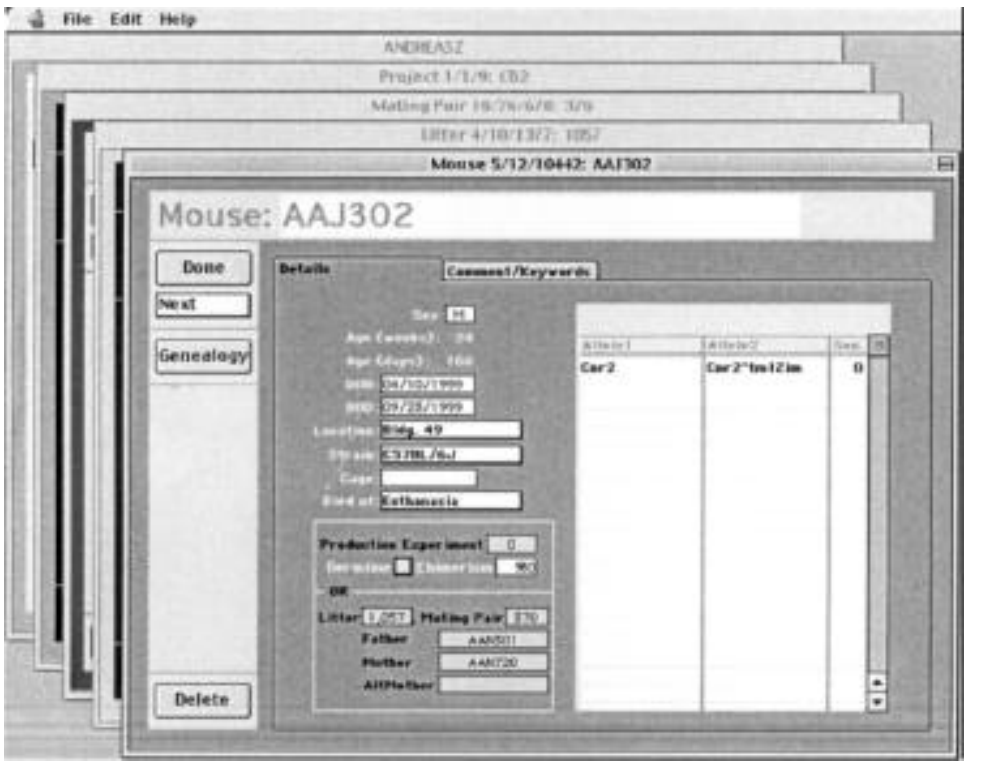

Figure 4. A Mouse record. User has "drilled down" from project CB2 through mating pair 370 and litter 1057 to the 5 th of 12 mice in the litter, of 10442 in the database. Founder mice are seen by descending the other branch of the hierarchy. The ID of the production experiment or mating pair/litter displayed here is not modifiable, nor are the calculated ages. Strain, Location and Cause of Death fields have bold outlines because they are pop-up choice lists - the short lists customized for this user or customized for this project, or the full (database-wide) lists, at the user's discretion. The Genealogy button will display a tree diagram that can be printed or saved as a text file to disk.

the user's selected records. Selection of mice can be made by any combination of expected search criteria such as age, sex, project, genotype, generation, percent chimerism, etc. An "Alert" function allows a researcher to define sets of search criteria, starting times/dates and time intervals, and the program performs those searches at those intervals, alerting the researcher to any records found that fit the criteria. This feature can be used to remind the researcher of pending tasks, for example, weaning litters.

Enhancements for version 3, which is currently under development, include making MouseBank accessible to the Web through Internet browsers, an automated timed-backup procedure so that the database administrator will not have to manually back up the data file and a selective-export feature so that departing researchers can take with them a copy of MouseBank and an exported data file that contains only their own project data. Other future enhancements include testing in the Windows environment, making MouseBank available to PC users, and the inclusion of a bar-coding scheme that will print a bar-coded cage card for each animal, allowing caretakers to update the database from a client computer in the animal facility by simply scanning the barcoded cage card with a reader wand. Eventually, as the technology drops in price, we hope to adopt a system of machine-readable, magnetically encoded ear or tail tags to automate mouse identification, reduce labor and minimize the potential for human error.

MouseBank is in the public domain and available to interested individuals or organizations free of charge. Three forms of MouseBank are available online at the National Institute of Mental Health Web site at http://rsb.info.nih. gov/mousebank/. Two require 4D 6.5.5 (a free upgrade from an earlier version of 4D 6.5 is available at the 4D Web site at http://www.4d.com): MouseBank 2.1.source is the source code file, which runs relatively slowly but can be modified by any 4D programmer. MouseBank 2.1 is a compiled file that is very fast but not modifiable. The third form, MouseBank 2.1.app, is a standalone version that, because it does not use 4D, cannot be used in client/server mode.
MouseBank 2.1.app is an effective demo, however, and might be downloaded as an evaluation copy by those unsure of whether to invest in 4D. Also available is MouseBank 2.0 User's Manual in Adobe ${ }^{\circledR}$ PDF format. All files have been compressed with Stuffit ${ }^{\circledR}$ (Aladdin Systems, Watsonville, CA, USA) in Stuffit 3.x format.

\section{ACKNOWLEDGMENTS}

The commercial software used in this application development project, 4th Dimension ${ }^{\circledR}$, 4D Compiler ${ }^{\circledR}$ and 4D Server ${ }^{\circledR}$, are the products of $4 \mathrm{D}$, Inc. (formerly ACI US, Inc.), San Jose, CA, USA (http://www.4d.com).

Received 19 May 2000; accepted 4 October 2000.

\section{Address correspondence to:}

Richard Hopley 36 Convent Drive, MSC 4094 National Institutes of Health Bethesda, MD 20892-4094, USA e-mail: rlhopley@codon.nih.gov 